\title{
Survival analyses of postoperative lung cancer patients: an investigation using Japanese administrative data
}

\author{
Susumu Kunisawa, Kazuto Yamashita, Hiroshi Ikai, Tetsuya Otsubo and Yuichi Imanaka*
}

\begin{abstract}
Long-term survival rates of cancer patients represent important information for policymakers and providers, but analyses from voluntary cancer registries in Japan may not reflect the overall situation. In 2003, the Diagnosis Procedure Combination Per-Diem Payment System (DPC/PDPS) for hospital reimbursement was introduced in Japan; more than half of Japan's acute care beds are currently covered under this system. Administrative data produced under the DPC system include claims data and clinical summaries for each admission. Due to the large amount of data spanning multiple institutions, this database may have applications in providing a more general and inclusive overview of healthcare. Here, we investigate the use of administrative data for analyses of long-term survival in cancer patients. We analyzed postoperative survival in 7,064 patients with primary non-small cell lung cancer admitted to 102 hospitals between April 2008 and March 2013 using DPC data. Survival was defined at the last date of examination or discharge within the study period, and the event was mortality during the same period. Overall survival rates for different cancer stages were calculated using the Kaplan-Meier method. Additionally, survival rates of cancer patients at clinical stage IA were compared between low- and high-volume hospitals using the Log-rank test. Postoperative 5-year survival for patients at stage IA was $85.8 \%(95 \% \mathrm{Cl}=78.6 \%-93.0 \%)$. High-volume hospitals had higher survival rates than hospitals with lower volume. Our findings using large-scale administrative data were similar to previous clinical registry reports, showing potential applications as a new method in analyzing up-to-date healthcare information.
\end{abstract}

Keywords: Lung cancer; Survival analysis; Administrative data; Japan

\section{Background}

Survival rates of cancer patients are a major concern for both patients and physicians, and are often referred to when determining patient prognoses and care strategies.

Cancer registries at the national level support the study of cancer etiology and outcomes, including analyses of survival rates (McLaughlin et al. 2010). In the US, the National Program of Cancer Registries was established in 1992 and provides population-based surveillance. In Europe, the European Network of Cancer Registries (http://www.encr. $\mathrm{eu} /$ ) and the European Cancer Registry (http://www.eurocare.it/) have been promoting collaborations between cancer registries within Europe and the European Union for

\footnotetext{
* Correspondence: imanaka-y@umin.net

Department of Healthcare Economics and Quality Management, Graduate School of Medicine, Kyoto University, Yoshida Konoe-cho, Sakyo-ku, Kyoto City 606-8501, Kyoto, Japan
}

more than 20 years. While national cancer registrations are also conducted in Scandinavia and the UK, (Butler et al. 2013) population-based registries in other countries frequently cover only a small portion of the population (Butler et al. 2013). Among these, German registries have progressed to a coverage of 40\% in 2012 (Hiripi et al. 2012).

Cancer registrations in Japan are still in the process of development. There exists a population-based cancer registry (http://www.jacr.info/); a hospital-based cancer registry (http://ncc.ctr-info.com/); and "organ-based" registries administrated by various medical associations and organizations, such as the Japanese Joint Committee for Lung Cancer Registration (http://haigan-touroku.jp/). However, these registries possess several shortcomings: for example, the population-based cancer registry has been reported to contain omissions of cases, and survival analyses have not been conducted (Sobue et al. 2007). The hospital-based

\section{实}


registry predominantly includes only data from specialized hospitals, (Sobue et al. 2007). and registries managed by medical associations generally include only voluntary participations. All of these registry databases do not adhere to a single format, and there is no framework that allows for their simple integration (Sobue et al. 2007; Hirata et al. 2012). Furthermore, medical associations conduct and report highly detailed surveys, but tend to struggle with low respondent rates (Sawabata et al. 2011).

The ability to conduct survival analyses using largescale administrative data, such as healthcare claims databases, would provide valuable information on patient prognoses and treatment effectiveness for a population. In 2003, Japan introduced a hospital reimbursement system known as the Diagnosis Procedure Combination/ Per-Diem Payment System (DPC/PDPS). This system is characterized by a requirement for healthcare providers to generate DPC data for each patient per hospitalization for reimbursement purposes. DPC data are uniformly formatted and include not only claims data and procedures, but also summaries of patient clinical information such as principal diagnoses and activities of daily living (ADL); in the case of cancer patients, the data also include disease-specific information, such as whether the cancer is primary or recurrent and the TNM classification of malignant tumors. In this way, the DPC/PDPS is in effect a registry of sorts for cancer patients who receive treatment at these hospitals.

Relatively short-term indicators for acute diseases, such as 30-day mortality and in-hospital mortality, have been widely studied (Lee et al. 2013; Sasaki et al. 2013; Yamashita et al. 2013) and applied as quality indicators (The Joint Commission 2014). Long-term survival may also be considered as a possible measure to describe the quality of healthcare. Although it should be kept in mind that long-term survival can be influenced by various determinants including environmental factors, differences in patient survival rates among institutions and regions are a common concern and have been studied (Meyerhardt et al. 2003; Hebert-Croteau et al. 2005).

Research using administrative data may allow the investigation of hospitals that do not participate in any cancer registry as well as hospitals across the boundaries of existing registries. The majority of acute care hospitals in Japan are reimbursed under the DPC system: over 1,400 hospitals are managed under this system as of 2013, and constitute more than $50 \%$ of all hospital beds in Japan. The clinical information available in these databases has allowed researchers in Japan to analyze medical care at the national, regional, hospital, and individual levels. With the increasing use of such administrative data for analyses, we are now able to shed light on relatively small hospitals that have potentially played a considerably large role in Japanese healthcare, but have heretofore been unavailable for analysis.
In 1995, our department established the Quality Indicator/Improvement Project (QIP; http://med-econ.umin. ac.jp/QIP/) in order to improve quality of healthcare in participant hospitals through the development, analysis, and feedback of quality indicators. Hospitals voluntarily participate in this project, and there are more than 400 hospitals currently enrolled. These participant hospitals continuously provide DPC data to the QIP; this data is analyzed and the results are periodically reported in feedback to participant hospitals. As this project has involved the collection of data for a relatively long period, it has developed the capacity for long-term analyses.

In this study, we attempt to open a new vista to conduct survival analyses of cancer patients using administrative data, focusing our analysis on postoperative lung cancer patients. This sample was selected due to the high incidence of the disease and high mortality rate of the patients, (Ministry of Health, Labour and Welfare 2013) thereby making this one of the most important fields in health services research. Our analysis may reveal up-todate details of patient survival with relatively high external validity, as our sample includes hospitals not currently enrolled in any cancer registry. Additionally, we investigate if there are differences in long-term survival among hospitals according to patient volume; the volume-outcomes relationship has been demonstrated in previous studies, and we analyze if our database and methodology are able to obtain similar conclusions to these registry-based studies.

\section{Methods}

Data

In this retrospective cohort study, we analyzed the postoperative survival of non-small cell lung cancer patients using administrative data. The data were obtained from the DPC-formatted database administered by the QIP. This database is very different from those of clinical registries: registries usually collect specific data for predetermined purposes and these data are submitted intentionally for analysis. In contrast, the DPC database uses medical claims data, which are routinely produced for all medical services with the primary intended purpose of reimbursement. These claims data are collected and analyzed to detect the statuses and progress of patients (Figure 1).

Non-small cell lung cancer patients were identified using International Classification of Diseases, 10th revision (ICD-10) codes C34\$ combined with cancer-specific information from the DPC data. Patients were included in analysis if they had been discharged from QIP member hospitals between April 2008 and March 2013 with the complete data necessary for our study. Although DPC data are produced either for inpatients or for both inpatients and outpatients depending on hospital, we selected hospitals that had contributed both inpatient and outpatient data to the database. 


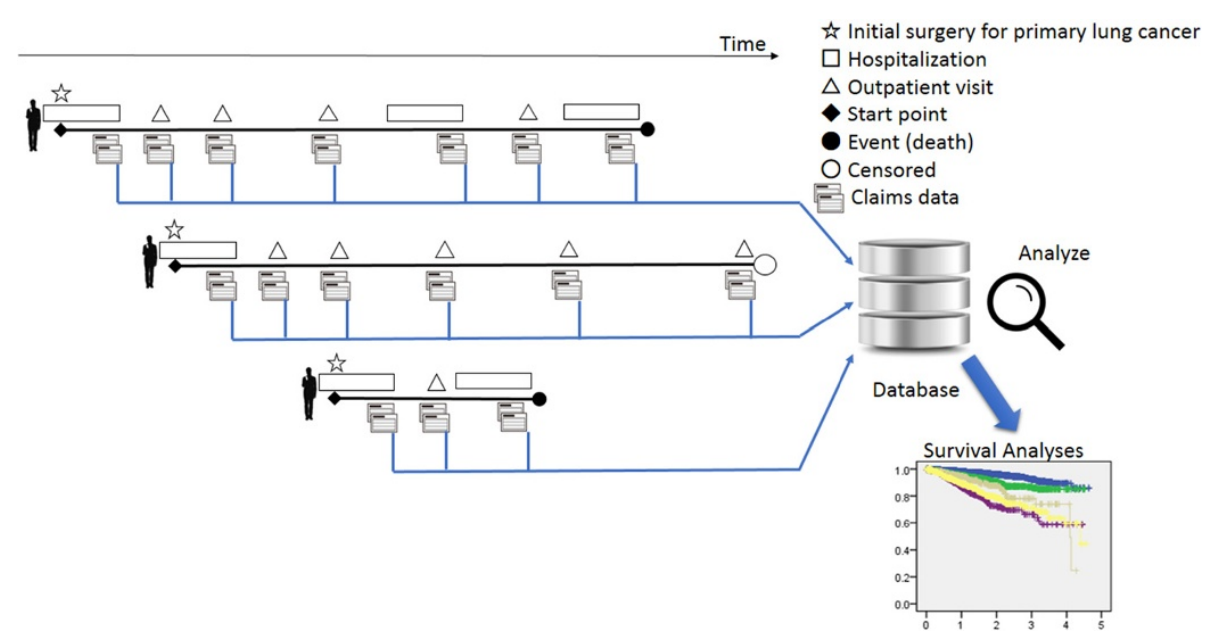

Figure 1 Data collection and analyses. All medical claims data produced for each hospitalization and subsequent outpatient visits are collected into a database, which is then analyzed in order to detect and trace statuses and progress of individual research subjects.

The details of operations for non-small cell lung cancer were identified from inpatient data. We selected patients who had undergone an operation for the first time for primary non-small cell lung cancer. We investigated patientlevel treatment histories from both the inpatient and outpatient database, and identified the last date of medical care or examination for each patient. Survival time was defined as the duration from the date of the initial operation for non-small cell lung cancer to the last recorded date for any medical service provided in the same hospital. For the survival analysis, the event was defined as patients who had died on the last recorded date for medical services, and surviving patients at the last recorded date were regarded as right censored.

It should be noted that the treatment histories for a single patient could only be followed for treatments provided in the same hospital. Therefore, censored cases include cases lost to follow-up due to changes in hospital, in addition to censoring resulting from the end of the study period. For this study, we assumed that the majority of patients would obtain healthcare from the same hospital after operation, as follow-up care would likely be conducted by specialists who are familiar with the patients.

Data concerning the base characteristics of patients and clinical TNM classification of cancer were also analyzed. We reclassified the TNM classes according to overall stage grouping. Because the study period included a transitional period with regard to the shift from Union for International Cancer Control (UICC)-6 to UICC-7 classification, our analysis included an overlap of both types of classification. We identified and standardized equivalent stages from the different staging standards for analysis: for example, T2N0M0 was equivalent to Stage IB in UICC-6; but under the current UICC-7 classification, Stage IB is equivalent to T2aN0M0 and Stage IIA is equivalent to T2bN0M0. Thus, a classification of Stage IB in this study includes both T2N0M0 of UICC-6 classification and T2aN0M0 of UICC-7 classification. We selected patients at Stages IA-IIIA for analysis.

\section{Comparison of survival rates according to patient volume} In addition, we compared survival rates between highvolume and low-volume hospitals. The relationship between patient volume and quality of healthcare has been widely reported, but there remains a lack of consensus on the nature of the relationship (Merlino 2007; Luchtenborg et al. 2013; Howington et al. 2013). We divided hospitals into two groups: those that had performed 30 or more operations per year, and those with fewer than 29 operations per year. For ease of comparison, we limited this analysis to patients with non-small cell lung cancer of stage IA (T1N0M0 in both UICC-6 and UICC-7) and who were completely independent with regard to ADL (i.e., patients with a score of 100 points in the Barthel Index).

\section{Statistical analysis}

Overall survival time for the various cancer stages was calculated using the Kaplan-Meier estimator with 95\% confidence intervals (CIs).

To analyze the effect of patient volume on the survival of postoperative stage IA lung cancer patients, we utilized the Log-rank test. All statistical analyses were conducted using SPSS software, Version 20.0.0.2 (IBM Inc., Japan).

\section{Ethical standard}

Prior to the study, the study procedures were reviewed and approved (\#E553) by the ethics review committee of Kyoto University Graduate School of Medicine, and 
informed consent was waived. The study complied with the Ethical Guidelines for Epidemiological Research of the Japanese national government, which include guidelines on protecting patient anonymity, and all the necessary conditions were satisfied for informed consent to be waived.

\section{Results and discussion}

Results

Survival rates of postoperative non-small cell lung cancer patients

We analyzed 7,064 primary non-small cell lung cancer patients from 102 hospitals. The various survival rates
( 1 year to 5 years) in stage IA to IIIA patients are presented in Table 1 . The postoperative 5 -year survival rate for stage IA patients was $85.8 \%$ ( $95 \% \mathrm{CI}=78.6 \%$ - 93.0\%). There were substantially more cases with Stages IA and IB when compared with the other stages. For comparative purposes, Table 1 also shows survival rates from previous reports by the Japanese Joint Committee for Lung Cancer Registration (JJCLCR) in 2011 (Sawabata et al. 2011) and the International Association for the Study of Lung Cancer (IASLC) in 2009 (Tanoue and Detterbeck 2009). Figure 2 shows the Kaplan-Meier curves for overall survival.

Table 1 Clinical stage-specific survival rates (1 year to 5 years) of postoperative non-small cell lung cancer patients

\begin{tabular}{|c|c|c|c|c|c|c|c|c|c|c|c|c|c|c|c|}
\hline \multirow[b]{3}{*}{ Clinical stages (DPC data) } & \multirow{3}{*}{$\mathbf{N}$} & \multicolumn{14}{|c|}{ Survival rates (\%) and number of subjects at risk } \\
\hline & & \multicolumn{3}{|c|}{$1 Y$} & \multicolumn{3}{|c|}{$2 Y$} & \multicolumn{3}{|c|}{$3 Y$} & \multicolumn{3}{|c|}{$4 Y$} & \multicolumn{2}{|r|}{$5 Y$} \\
\hline & & $\%$ & $95 \% \mathrm{Cl}$ & $n^{a}$ & $\%$ & $95 \% \mathrm{Cl}$ & $\mathrm{n}$ & $\%$ & $95 \% \mathrm{Cl}$ & $\mathrm{n}$ & $\%$ & $95 \% \mathrm{Cl}$ & $\mathrm{n}$ & $\%$ & $95 \% \mathrm{Cl}$ \\
\hline $\mid A$ & 3812 & 98.2 & $( \pm 0.6)$ & 2014 & 96.3 & $( \pm 0.8)$ & 939 & 92.6 & $( \pm 1.8)$ & 318 & 89.3 & $( \pm 3.1)$ & 36 & 85.8 & $( \pm 7.3)$ \\
\hline IB & 1581 & 95.4 & $( \pm 1.2)$ & 781 & 90.7 & $( \pm 2.2)$ & 365 & 86.6 & $( \pm 2.9)$ & 137 & 85.0 & $( \pm 3.7)$ & 25 & 85.0 & $( \pm 3.7)$ \\
\hline$\| \mathrm{A}$ & 420 & 94.6 & $( \pm 2.5)$ & 200 & 87.8 & $( \pm 4.7)$ & 84 & 78.0 & $( \pm 8.0)$ & 21 & 73.9 & $( \pm 11.0)$ & 4 & 24.6 & $( \pm 39.6)$ \\
\hline$\| B$ & 480 & 86.4 & $( \pm 3.7)$ & 230 & 71.7 & $( \pm 5.9)$ & 92 & 66.3 & $( \pm 7.6)$ & 34 & 58.6 & $( \pm 10.6)$ & 7 & 58.6 & $( \pm 10.6)$ \\
\hline IIIA & 771 & 89.1 & $( \pm 2.5)$ & 386 & 78.7 & $( \pm 4.1)$ & 146 & 71.1 & $( \pm 6.1)$ & 58 & 59.3 & $( \pm 11.6)$ & 12 & 44.5 & $( \pm 26.7)$ \\
\hline \multicolumn{16}{|c|}{ JJCLCR (2011) Clinical stages 6th edition ${ }^{\text {b }}$} \\
\hline IA & 6295 & 97.0 & & & 92.7 & & & 89.1 & & & 85.5 & & & 82.0 & \\
\hline IB & 2788 & 91.0 & & & 81.9 & & & 74.8 & & & 68.0 & & & 63.4 & \\
\hline$\| \mathrm{A}$ & 203 & 89.7 & & & 75.0 & & & 64.3 & & & 59.2 & & & 55.4 & \\
\hline$\| \mathrm{B}$ & 899 & 83.7 & & & 69.5 & & & 59.8 & & & 54.0 & & & 48.6 & \\
\hline IIIA & 940 & 80.9 & & & 64.3 & & & 53.6 & & & 47.7 & & & 43.3 & \\
\hline \multicolumn{16}{|c|}{ JJCLCR (2011) Clinical stages 7th edition ${ }^{b}$} \\
\hline IA & 6295 & 97.0 & & & 92.7 & & & 89.1 & & & 85.5 & & & 82.0 & \\
\hline IB & 2339 & 92.5 & & & 84.4 & & & 77.6 & & & 70.8 & & & 66.1 & \\
\hline$\| \mathrm{A}$ & 819 & 88.7 & & & 85.4 & & & 66.8 & & & 60.2 & & & 54.5 & \\
\hline$\| \mathrm{B}$ & 648 & 80.0 & & & 63.6 & & & 54.7 & & & 50.4 & & & 46.4 & \\
\hline IIIA & 1216 & 81.4 & & & 64.7 & & & 53.7 & & & 47.3 & & & 42.8 & \\
\hline \multicolumn{16}{|c|}{ IASLC (2009) Clinical stages 6th edition ${ }^{c}$} \\
\hline $\mid \mathrm{A}$ & 831 & & & & & & & & & & & & & 50 & \\
\hline IB & 1842 & & & & & & & & & & & & & 40 & \\
\hline$\| \mathrm{A}$ & 25 & & & & & & & & & & & & & 24 & \\
\hline$\| \mathrm{B}$ & 2151 & & & & & & & & & & & & & 25 & \\
\hline IIIA & 3005 & & & & & & & & & & & & & 18 & \\
\hline \multicolumn{16}{|c|}{ IASLC (2009) Clinical stages 7th edition ${ }^{c}$} \\
\hline IA & 831 & & & & & & & & & & & & & 50 & \\
\hline IB & 1284 & & & & & & & & & & & & & 43 & \\
\hline$\| \mathrm{A}$ & 483 & & & & & & & & & & & & & 36 & \\
\hline$\| B$ & 2248 & & & & & & & & & & & & & 25 & \\
\hline IIIA & 3175 & & & & & & & & & & & & & 19 & \\
\hline
\end{tabular}

${ }^{\mathrm{a}}$ Number at risk.

${ }^{b}$ Data from the Japanese Joint Committee for Lung Cancer Registration (JJCLCR) in 2011 (Sawabata et al. 2011); 95\% CI were not available for these data.

'Data from the International Association for the Study of Lung Cancer (IASLC) in 2009 (Tanoue and Detterbeck 2009); 95\% CI were not available for these data. 


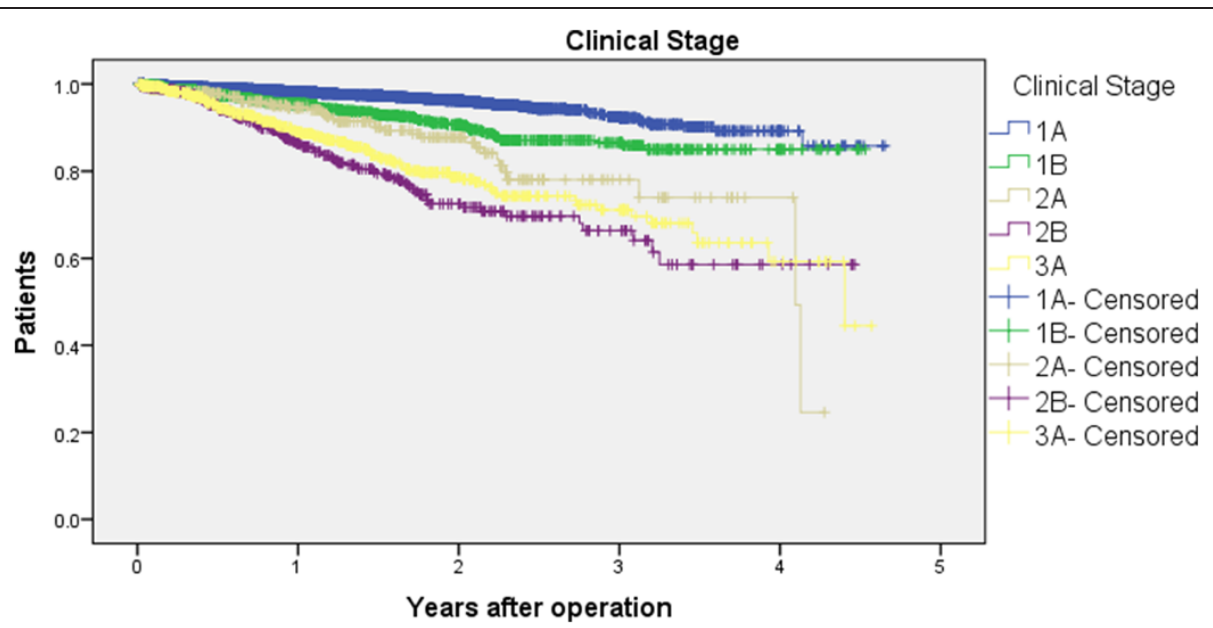

Figure 2 Survival curves according to clinical cancer stage. Survival curves according to clinical cancer stage for surgically managed primary non-small cell lung cancer patients.

\section{Comparison of survival rates according to patient volume} For the analysis of survival according to patient volume, the sample consisted of 3,379 patients at clinical stage IA with complete independence in terms of ADL. The highvolume hospital group comprised 33 hospitals with 2,587 cases, and the low-volume hospital group comprised 63 hospitals with 792 cases. The base characteristics of these patients are shown in Table 2, and Chi-square tests revealed no significant differences $(P>0.05)$ in gender and ages between the two hospital groups. The effect of patient volume on survival was assessed using the Log-rank test for the Kaplan-Meier survival curves. Table 3 and Figure 3 show the survival rates of each group. The 5-year survival rates for high-volume hospitals and low-volume hospitals were $92.1 \pm 2.9 \%$ and $81.4 \pm 11.1 \%$, respectively; the results showed a significant difference between the groups $(\mathrm{p}=0.05)$.

\section{Discussion}

In this study, we conducted postoperative survival analyses of lung cancer patients using an administrative

Table 2 Base characteristics of patients admitted to low-volume hospitals $(n=63)$ and high-volume hospitals ( $n=33)$

\begin{tabular}{lccc}
\hline & & Low-volume hospitals & High-volume hospitals \\
\hline Gender & Male & 467 & 1463 \\
& Female & 325 & 1124 \\
Age & $<65$ y & 246 & 857 \\
& $65-74$ y & 288 & 986 \\
& $\geq 75$ y & 258 & 744 \\
Death & 29 & 52 \\
Total & & 792 & 2587 \\
\hline
\end{tabular}

Values in the table indicate numbers of patients. database. As shown in Figure 2, survival rates varied substantially among the clinical stages. The findings were similar to those in previous Japanese reports that utilized registry data, (Sawabata et al. 2011) which supports the applicability of our administrative data-based method. The second analyses showed that our findings corroborate the results from previous studies in which higher volume hospitals showed a higher survival rate.

The contribution of this study to the field lies in the use of large-scale administrative data to analyze longterm postoperative survival. The comparably larger sample sizes for Stages IA and IB strengthen the validity of our findings; however, the sample sizes were smaller in later-stage cancer, and the details of survival rates for these groups were more difficult to discern. The survival rates for the two early stages were very similar to those previously reported in Japan, (Sawabata et al. 2011) which supports the applicability of this method, although it should be noted that the figures were very different from those in an international report (Tanoue and Detterbeck 2009). Survival rates in this study were slightly higher than those previously reported in Japan (Tanoue and Detterbeck 2009). This discrepancy may be the result of a possible bias in our study due to the loss to follow-up of patients who had died in a different hospital from the initial hospital where the operation was conducted, which is discussed in further detail below. Alternatively, the differences in results may be due to the differences in composition of the study samples, wherein our analysis may have contained more general hospitals than analyses using only hospitals enrolled in cancer registries. Also, the differences in study periods between our analysis and previous studies may have contributed to the observed differences in survival rates. Our study comprises up-to-date data, and may have 
Table 3 Survival in surgically managed primary non-small cell lung cancer patients with a clinical stage of IA categorized by patient volume per hospital

\begin{tabular}{|c|c|c|c|c|c|c|c|c|c|c|c|c|c|c|c|}
\hline \multirow[b]{3}{*}{ Patient Volume } & \multirow{3}{*}{$\mathrm{N}$} & \multicolumn{14}{|c|}{ Survival rates (\%) and number of subjects at risk } \\
\hline & & \multicolumn{3}{|c|}{$1 \mathrm{Y}$} & \multicolumn{3}{|c|}{$2 Y$} & \multicolumn{3}{|c|}{$3 Y$} & \multicolumn{3}{|c|}{$4 Y$} & \multicolumn{2}{|c|}{$5 Y$} \\
\hline & & $\%$ & $95 \% \mathrm{Cl}$ & $\mathrm{n}^{\mathrm{a}}$ & $\%$ & $95 \% \mathrm{Cl}$ & $\mathrm{n}^{\mathrm{a}}$ & $\%$ & $95 \% \mathrm{Cl}$ & $\mathrm{n}^{\mathrm{a}}$ & $\%$ & $95 \% \mathrm{Cl}$ & $\mathrm{n}^{\mathrm{a}}$ & $\%$ & $95 \% \mathrm{Cl}$ \\
\hline High-volume Hospitals & 2587 & 98.5 & $( \pm 0.6)$ & 1336 & 97.3 & $( \pm 0.5)$ & 614 & 93.9 & $( \pm 1.1)$ & 195 & 92.1 & $( \pm 2.9)$ & 3 & 92.1 & $( \pm 2.9)$ \\
\hline Low-volume Hospitals & 792 & 97.8 & $( \pm 1.2)$ & 427 & 94.9 & $( \pm 2.4)$ & 163 & 90.6 & $( \pm 4.5)$ & 50 & 81.4 & $( \pm 11.1)$ & 18 & 81.4 & $( \pm 11.1)$ \\
\hline
\end{tabular}

${ }^{a}$ Number at risk.

benefited from general progress in medical care. Further studies are needed to clarify these differences.

The data used in our analysis were only obtained from the administrative data submitted by each hospital, and were not merged with external data sources such as population databases or registration databases. Database integration in Japan presents considerable difficulties due to a lack of national unique identification data, such as social security numbers or national insurance numbers. DPC data may represent a tool to overcome these difficulties, as hospitals under the DPC/PDPS encompass more than half of all acute care beds in Japan, and treat approximately $90 \%$ of all acute inpatients (Murata et al. 2013). As DPC data are uniformly formatted, quick multi-institutional analyses are possible and can provide valuable up-to-date information for policymakers, healthcare providers, and the general public.

Hospitals with different patient volumes were found to have different survival rates in our study, which was compatible with previous reports wherein higher volume hospitals showed more favorable outcomes (Merlino 2007; Luchtenborg et al. 2013; Howington et al. 2013). Furthermore, as DPC data contain some clinical information, risk adjustment is possible. Owing to prompt analysis, the use of these data may allow long-term survival to be established as a quality indicator, with applications in comparing regional variations or identifying hospitals with exceptionally low survival rates.

Another point worth noting is that this method can be adapted to other patient populations, such as for other cancers and other diseases. Furthermore, this type of study may have applications in countries other than Japan. Japanese DPC data may serve as a model when designing or reforming other insurance claims databases, as this system not only enables analyses of in-hospital situations, but also provides useful medical data for analyzing patients after being discharged.

Another novelty of this study is that owing to the extensive database, we were able to deal with relatively large sample sizes for each analysis. The benefits of large-scale analysis were demonstrated in the narrow 95\% CIs; even the CIs around 5-year survival-which was the full time period of our study-were considerably narrow, indicating a level of reliability in our findings. In other words, we were able to fully utilize the inpatient data spanning a 5-year period by evaluating 5-year survival rates. This was largely due to the use of the Kaplan-Meier method. When the sample size is relatively small, researchers need to wait for many years to obtain a sufficient sample size for an analysis: for example, several years may be required to enroll sufficient cases and several more years to conduct observations

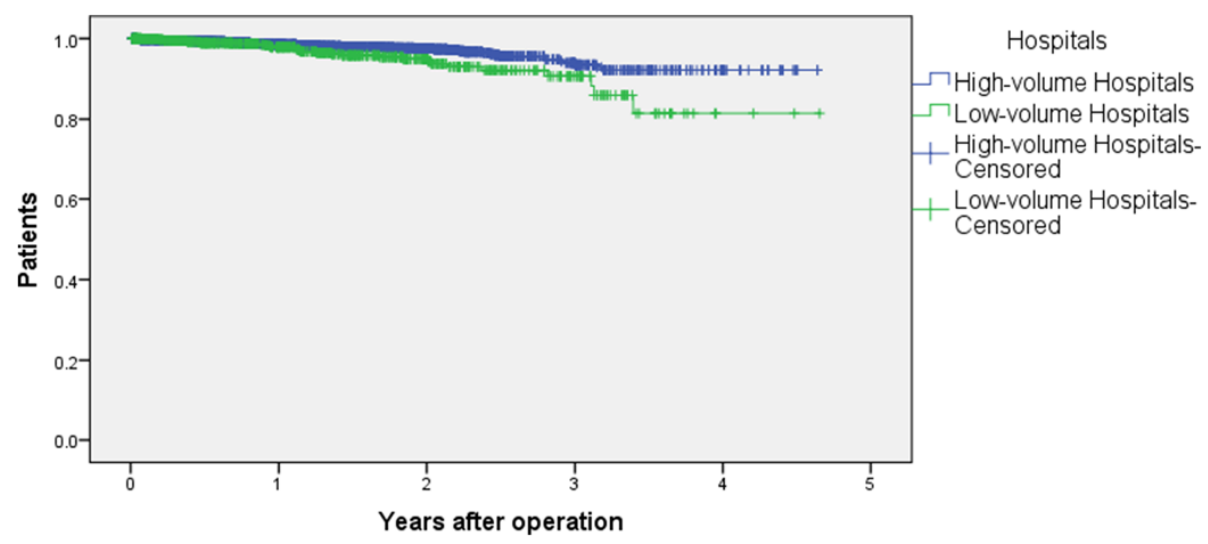

Figure 3 Survival and patient volume per hospital. Survival curves by patient volume per hospital for surgically managed primary non-small cell lung cancer patients with a clinical stage of IA. 
after the last enrollment, leading to a lengthy data collection period. However, the DPC data allow quick and up-to-date insight into patient survival over several years, which can support medical staff and policymakers in monitoring current cancer treatments and determining appropriate responses. For example, comparisons of survival rates among different regions may indicate a necessity to re-formulate current healthcare plans.

A limitation of this study is that the administrative data do not include extensive clinical information. For example, DPC data do not contain information regarding pathological TNM stages and pathological types, which are provided in current cancer registries' reports. Other clinical factors such as comorbidities and cotreatments may influence patient survival, and their effects should also be investigated in future analyses. Therefore, we are unable to make definitive conclusions regarding the effects of hospital case volumes on survival without accounting for such factors. However, our results were compatible with those from previous reports. As our method provides the ability to rapidly assess overall situations, DPC data collection criteria can be expanded in the future to include more clinical information if deemed necessary for analysis. This would, however, require the need to weigh the time and effort in implementing additional data collection against its benefits.

Next, the analyses were limited to tracking data from one hospital for each patient. The starting date for analysis of each patient was the initial surgery date for lung cancer at a DPC hospital. Therefore, there may be time lapses between the initial diagnosis of cancer and the initial treatment. We were unable to determine if a patient had obtained treatment at other hospitals prior to our analysis, as the data could not be interlinked among the various institutions. However, the vast majority of treatment for cancer would likely be conducted in specialized medical institutions, and our method therefore still has validity in investigating cancer epidemiology in Japan. Similarly, the data only allow the tracking of patient care within the same hospital, and patients who subsequently seek healthcare at other institutions are lost to followup. Thus, the survival rates reported in this study may be higher than the actual survival rates, as some patients may have sought healthcare or died in other hospitals or hospices. We, at this time, assume that most postoperative patients for lung cancer are followed up at the same hospital where they had undergone surgery, and the majority of recurrent cases or patients with deteriorating conditions are likely to be treated at the same hospital. In addition, some patients may travel a relatively long distance from their homes to undergo specialized surgery, but receive follow-up care at a closer regional hospital. As such cases would also be lost to follow-up, future studies that focus on patients residing in the same region as the hospital where they receive care may reduce this loss of data.

It should be emphasized that there is a need for databases composed of public data to be systematically designed with the intrinsic capability to integrate with other databases in order to maximize their contributions and reduce loss to follow-up. The limitations of the data source used in this study arose from the lack of integration across Japanese public databases. The importance of this study lies in the finding that routinely collected medical claims data have applications in long-term survival analyses. While registry databases are undeniably useful, the integration of other large databases, such as those comprising claims data, should be considered in the analysis of any healthcare topic. Although further studies are needed to improve the follow-up ability of the data and reduce possible bias due to the aforementioned limitations, the integration of data sources is an essential step for improving health services research. In order to both strengthen the validity of analyses similar to this study and reinforce registry databases, public database systems should be designed to allow for integration with other databases. We believe that the further discovery and validation of effective applications of administrative claims databases would support the provision of up-to-date information to help improve healthcare quality and outcomes.

\section{Conclusions}

We conducted survival analyses of postoperative nonsmall cell lung cancer patients using a Japanese administrative claims database, which covered a wide range of hospitals that included providers not enrolled in any cancer registry. Our findings were consistent with previous reports using analyses of registries, showing that this methodology may have applications as a useful outcomes measurement tool for medical staff and policymakers.

\section{Abbreviations}

(DPC/PDPS): Diagnosis Procedure Combination Per-Diem Payment System; (ADL): Activities of daily living; (ICD-10): International Classification of Diseases, 10th revision; (QIP): Quality Indicator/Improvement Project; (UICC): Union for International Cancer Control; (CI): Confidence interval; (JJCLCR): Japanese Joint Committee for Lung Cancer Registration; (IASLC): International Association for the Study of Lung Cancer.

\section{Competing interests}

The authors declare that they have no competing interests.

\section{Authors' contributions}

SK, and $\mathrm{YI}$ conceived and designed the study. $\mathrm{YI}$ and $\mathrm{HI}$ acquired the data. SK, KY, HI, TO and YI analyzed and interpreted the data. SK drafted the manuscript, and all authors contributed substantially to its revision. All authors have read and approved the submitted manuscript. The manuscript has not been submitted elsewhere nor published elsewhere in whole. The manuscript has been carefully reviewed by an experienced editor whose first language is English and who specializes in editing papers written by scientists whose native language is not English. YI takes responsibility for the paper as a whole. 


\section{References}

Butler J, Foot C, Bomb M, Hiom S, Coleman M, Bryant H, Vedsted P, Hanson J, Richards M, The ICBP Working Group (2013) The International Cancer Benchmarking Partnership: an international collaboration to inform cancer policy in Australia, Canada, Denmark, Norway, Sweden and the United Kingdom. Health Policy 112(1-2):148-155

Hebert-Croteau N, Brisson J, Lemaire J, Latreille J, Pineault R (2005) Investigating the correlation between hospital of primary treatment and the survival of women with breast cancer. Cancer 104(7):1343-1348

Hirata K, Okita K, Imamura M, Ohta S, Kukita K, Kyuno D, Mizuguchi T, Furuhata T (2012) Expansion and assessment of cancer treatment guidelin based on the database indicator on cancer registry (in Japanese). Surgery Frontier 19(4):37-44

Hiripi E, Gondos A, Emrich K, Holleczek B, Katalinic A, Luttmann S, Sirri E, Brenner H, GEKID Cancer Survival Working Group (2012) Survival from common and rare cancers in Germany in the early 21st century. Ann Oncol 23(2):472-479

Howington JA, Blum MG, Chang AC, Balekian AA, Murthy SC (2013) Treatment of stage I and II non-small cell lung cancer: diagnosis and management of lung cancer, 3rd edn: American College of Chest Physicians evidence-based clinical practice guidelines. Chest 143(5 Suppl):e278S-e313S

Lee J, Morishima T, Kunisawa S, Sasaki N, Otsubo T, Ikai H, Imanaka Y (2013) Derivation and validation of in-hospital mortality prediction models in ischaemic stroke patients using administrative data. Cerebrovasc Dis 35(1):73-80

Luchtenborg M, Riaz SP, Coupland VH, Lim E, Jakobsen E, Krasnik M, Page R, Lind MJ, Peake MD, Moller H (2013) High procedure volume is strongly associated with improved survival after lung cancer surgery. J Clin Oncol 31(25):3141-3146

McLaughlin RH, Clarke CA, Crawley LM, Glaser SL (2010) Are cancer registries unconstitutional? Soc Sci Med 70(9):1295-1300

Merlino J (2007) Defining the volume-quality debate: is it the surgeon, the center or the training? Clin Colon Rectal Surg 20(3):231-236

Meyerhardt JA, Catalano PJ, Schrag D, Ayanian JZ, Haller DG, Mayer RJ, Macdonald JS, Benson AB III, Fuchs CS (2003) Association of hospital procedure volume and outcomes in patients with colon cancer at high risk for recurrence. Ann Intern Med 139(8):649-657

Ministry of Health, Labour and Welfare (2013) Heisei 24 nen jinko dotai tokei (Population survey report in 2012) (in Japanese)., Available: http://www.mhlw. go.jp/toukei/saikin/hw/jinkou/geppo/nengai12/dl/kekka.pdf [2014, April 16]

Murata A, Okamoto K, Matsuda S, Kuwabara K, Ichimiya Y, Matsuda Y, Kubo T, Fujino Y (2013) Multivariate analysis of factors influencing length of hospitalization and medical costs of cholecystectomy for acute cholecystitis in Japan: a national database analysis. Keio J Med 62(3):83-94

Sasaki N, Lee J, Park S, Umegaki T, Kunisawa S, Otsubo T, Ikai H, Imanaka Y (2013) Development and validation of an acute heart failure-specific mortality predictive model based on administrative data. Can J Cardiol 29(9):1055-1061

Sawabata N, Fujii Y, Asamura H, Nomori H, Nakanishi Y, Eguchi K, Mori M, Okumura M, Miyaoka E, Yokoi K (2011) Analysis of lung cancer registry cases resected in 2004 Japanese Joint Committee for Lung Cancer Registration (in Japanese with English abstract). J Jpn Assoc Chest Surg 25(1):107-123

Sobue T, Tsukuma H, Okamoto N, Ajiki W (2007) Handbook on Population-Based Cancer Registration in Japan Fifth Revision (in Japanese)., Available: http://ncrp.ncc.go.jp/file/seibi/tebiki/tebiki_zentai.pdf

Tanoue LT, Detterbeck FC (2009) New TNM classification for non-small-cell lung cancer. Expert Rev Anticancer Ther 9(4):413-423

The Joint Commission (2014) Performance Measurement and S3 for Hospitals. Available: http://www.jointcommission.org/accreditation/ performance_measurementoryx.aspx [2014, April 16]

Yamashita K, Ikai H, Nishimura M, Fushimi K, Imanaka Y (2013) Effect of certified training facilities for intensive care specialists on mortality in Japan. Crit Care Resusc 15(1):28-32

doi:10.1186/2193-1801-3-217

Cite this article as: Kunisawa et al: Survival analyses of postoperative lung cancer patients: an investigation using Japanese administrative data. SpringerPlus 2014 3:217.

\section{Submit your manuscript to a SpringerOpen ${ }^{\odot}$ journal and benefit from:}

- Convenient online submission

- Rigorous peer review

- Immediate publication on acceptance

- Open access: articles freely available online

- High visibility within the field

- Retaining the copyright to your article

Submit your next manuscript at $>$ springeropen.com 